\title{
Incidence and risk factors associated with early death in patients with emergency department septic shock
}

\author{
Matthew S. Reaven ${ }^{1}$, Nigel L. Rozario ${ }^{2}$, Maggie S. J. McCarter ${ }^{2}$, Alan C. Heffner ${ }^{3,4}$ \\ ${ }^{1}$ Division of Pulmonary, Allergy, Critical Care, and Sleep Medicine, Department of Medicine, Emory University School of Medicine, Atlanta, GA; ${ }^{2}$ Center for \\ Outcomes Research and Evaluation, Atrium Health, Charlotte, NC; Departments of ${ }^{3}$ Internal Medicine and ${ }^{4}$ Emergency Medicine, Carolinas Medical Center, \\ Charlotte, NC, USA
}

Background: Limited research has explored early mortality among patients presenting with septic shock. The objective of this study was to determine the incidence and factors associated with early death following emergency department (ED) presentation of septic shock.

Methods: A prospective registry of patients enrolled in an ED septic shock clinical pathway was used to identify patients. Patients were compared across demographic, comorbid, clinical, and treatment variables by death within 72 hours of ED presentation.

Results: Among the sample of 2,414 patients, overall hospital mortality was $20.6 \%$. Among patients who died in the hospital, mean and median time from ED presentation to death were 4.96 days and 2.28 days, respectively. Death at 24,48 , and 72 hours occurred in 5.5\%, 9.5\%, and $11.5 \%$ of patients, respectively. Multivariate regression analysis demonstrated that the following factors were independently associated with early mortality: age (odds ratio $[\mathrm{OR}], 1.04 ; 95 \%$ confidence interval $[\mathrm{Cl}], 1.03-1.05)$, malignancy $(\mathrm{OR}, 1.53 ; 95 \% \mathrm{Cl}, 1.11-2.11)$, pneumonia $(\mathrm{OR}, 1.39 ; 95 \% \mathrm{Cl}$, $1.02-1.88)$, urinary tract infection $(\mathrm{OR}, 0.63 ; 95 \% \mathrm{Cl}, 0.44-0.89)$, first shock index $(\mathrm{OR}, 1.85 ; 95 \%$ $\mathrm{Cl}, 1.27-2.70)$, early vasopressor use $(\mathrm{OR}, 2.16 ; 95 \% \mathrm{Cl}, 1.60-2.92)$, initial international normalized ratio $(\mathrm{OR}, 1.14 ; 95 \% \mathrm{Cl}, 1.07-1.27)$, initial albumin $(\mathrm{OR}, 0.55 ; 95 \% \mathrm{Cl}, 0.44-0.69)$, and first serum lactate (OR, 1.21; $95 \% \mathrm{Cl}, 1.16-1.26)$.

Conclusions: Adult septic shock patients experience a high rate of early mortality within 72 hours of ED arrival. Recognizable clinical factors may aid the identification of patients at risk of early death.

Key Words: early mortality; emergency department; sepsis; septic shock

\section{INTRODUCTION}

Septic shock is one of the most common reasons for intensive care unit (ICU) admission, with up to 750,000 cases per year in the United States [1]. Despite treatment advances, septic shock is a leading cause of death, with hospital mortality rates ranging from $20 \%$ to $45 \%[2,3]$. Our current treatment paradigm emphasizes early recognition and treatment, but the vast majority of previous research focused on mortality within the hospital or over longer time periods. This makes it difficult to understand mortality directly related to refractory shock compared

\section{Original Article}

Received: June 23, 2021

Revised: October 8, 2021

Accepted: November 8, 2021

\section{Corresponding author}

Matthew S. Reaven

Department of Medicine, Emory University School of Medicine, 615 Michael St, Suite 205, Atlanta, GA 30322, USA

Tel: +1-330-327-7840

E-mail:mreaven@emory.edu
Copyright () 2022 The Korean Society of Critical Care Medicine

This is an Open Access article distributed under the terms of Creative Attributions Non-Commercial License (https://creativecommons.org/li-censes/by-nc/4.0/) which permits unrestricted noncommercial use, distribution, and reproduction in any medium, provided the original work is properly cited. 
to that related to unremitting organ dysfunction and persistent debility following initial stabilization or recovery, including mortality related to secondary injuries such as nosocomial infection.

Early sepsis mortality is an uncommon focus of prior investigations. Specifically, few investigations have described the prevalence of early mortality or factors associated with early mortality. The objective of this study was to characterize the rate of early death following emergency department (ED) admission for septic shock and to determine clinical factors associated with this outcome.

\section{MATERIALS AND METHODS}

This study was approved by Institutional Review Board of Atrium Health (IRB No. IRB00001088), and the requirement for patient consent was waived.

A prospective quality improvement registry of patients enrolled in our healthcare system's ED septic shock clinical pathway was used to identify patients. Patients enrolled in five urban metropolitan hospitals, including a large tertiary referral center, from January 2014 to May 2018, were included in the study. Criteria for patient entry into our high-risk sepsis pathway were the presence of suspected infection plus at least one of the following: hypotension despite $20 \mathrm{ml} / \mathrm{kg}$ intravenous fluid defined as either systolic blood pressure (SBP) less than $90 \mathrm{~mm}$ $\mathrm{Hg}$ or mean arterial pressure (MAP) less than $65 \mathrm{~mm} \mathrm{Hg}$, or a serum lactate level greater than or equal to $4 \mathrm{mmol} / \mathrm{L}$. Among registered patients, we excluded those without discharge diagnoses consistent with infection, sepsis, severe sepsis or septic shock. Patients were excluded if they were not admitted to the ICU, with this requirement intended to represent intention for aggressive therapy for acute critical illness. While the decision to admit a patient to the ICU is ultimately at the discretion of the ICU physician, at our institutions it is standard practice to admit septic shock patients to the ICU for close monitoring and aggressive treatment unless the patient's goals of care state otherwise. Lastly, patients transferred between ICUs and patients from hospitals with less than 30 entries per year were excluded. Figure 1 details the exclusion criteria.

\section{Variable Definitions}

ED presentation time was defined by first ED vital signs. Shock index (SI) was calculated based on heart rate divided by SBP. Early vasopressor use was defined by initiation within 6 hours of ED presentation. Patients were compared across demo-

\section{KEYMESSAGES}

- A significant focus is placed on early recognition and treatment of sepsis.

- Few studies evaluate early mortality (within 72 hours of presentation).

- Several identifiable factors have been shown to correlate with early mortality.

- Identifying patients at high risk of early mortality may aid in their management.

graphic, comorbid, clinical, and treatment variables based on whether they were dead or alive 72 hours from ED triage. Descriptive statistics in the form of median and interquartile range were calculated for continuous variables and frequencies and percentages were calculated for categorical variables. The Wilcoxon signed-rank test was used to compare continuous variables between the 72-hour death group and the non72-hour death group. Similarly, the chi-square or Fisher's exact test were used to compare categorical variables between groups.

A Kaplan-Meier curve was plotted showing survival rate relative to hours from ED triage. Two receiver operating curves

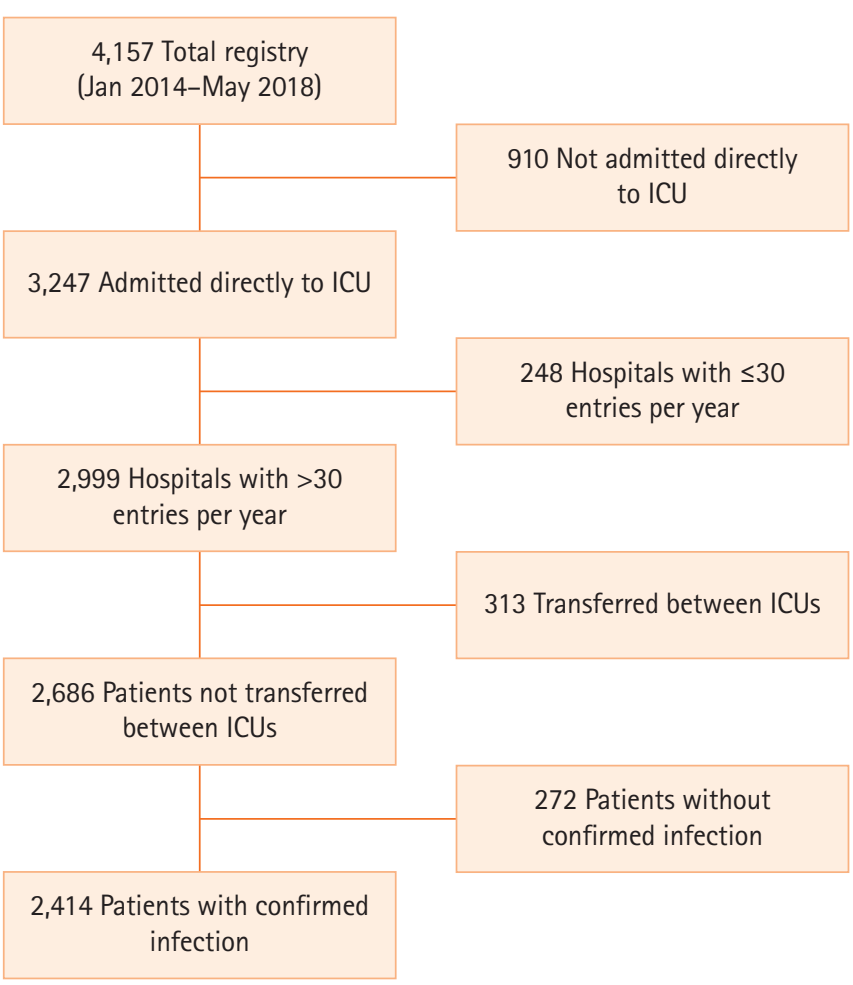

Figure 1. Flow diagram of patient selection. ICU: intensive care unit. 
(ROC) were plotted, one for initial lactate and one for initial SI to predict 72-hour mortality, and the area under the curve (AUC) and optimal cut points for each were calculated. After clinical consideration and assessment of statistically significant factors predicting early mortality in univariate analysis, a final logistic model was used to assess predictive variables for early mortality.

\section{RESULTS}

During the study period, a total of 4,157 patients were registered in our ED septic shock clinical pathway. Of this group, $2,414(58.1 \%)$ met inclusion criteria for this investigation and comprised our study population. Baseline demographic and clinical variables of study subjects are described in Table 1 . Among these patients, 1,241 (51.4\%) were male, 1,667 (69.1\%) were white, 601 (24.9\%) were African-American, 78 (3.2\%) were Hispanic, and 31 (1.3\%) were Asian. The average age was 64.5 years. Major comorbidities included heart failure (24.4\%), chronic kidney disease (23.8\%), acute kidney injury (31.0\%), cirrhosis (12.2\%), chronic obstructive pulmonary disease (27.3\%), diabetes mellitus (40.2\%), malignancy (20.7\%), and dementia (19.9\%). The most common source of infection was pulmonary $(29.7 \%)$ followed by urinary tract $(27.9 \%)$.

Overall study group hospital mortality was $20.6 \%$ (95\% confidence interval [CI], 19.0\%-22.2\%). Among those dying in the hospital, mean and median time from ED presentation to death were 4.96 days (95\% CI, 4.41-5.51 days) and 2.28 days (interquartile range, 0.88-7.22 days) respectively. Cumulative death at 24,48 , and 72 hours occurred in $5.5 \%$ (95\% CI, 4.7\%-6.5\%), 9.5\% (95\% CI, 8.4\%-10.8\%), and $11.5 \%$ (95\% CI, $10.3 \%-12.9 \%)$, respectively. Figure 2 demonstrates the surviv-

Table 1. Baseline patient group characteristics

\begin{tabular}{|c|c|c|c|c|}
\hline Factor & Total $(n=2,414)$ & Non-72-hr death $(n=2,136)$ & 72-hr death $(n=278)$ & P-value \\
\hline \multicolumn{5}{|l|}{ Demographics } \\
\hline Age (yr) & $66.1(54.4-76.4)$ & $65.3(53.6-75.4)$ & $71.4(61.0-81.4)$ & $<0.001^{\mathrm{a}}$ \\
\hline BMI $\left(\mathrm{kg} / \mathrm{m}^{2}\right)$ & $26.5(22.3-31.8)$ & $26.7(22.5-32.0)$ & $25.1(20.9-30.4)$ & $<0.001^{a}$ \\
\hline Male & $1,241(51.4)$ & $1,091(51.1)$ & $150(54.0)$ & $0.370^{6}$ \\
\hline Dementia & 481 (19.9) & 412 (19.3) & $69(24.8)$ & $0.030^{b}$ \\
\hline Malignancy & $500(20.7)$ & 413 (19.3) & $87(31.3)$ & $<0.001^{b}$ \\
\hline DNR status & $232(9.6)$ & $193(9.0)$ & $39(14.0)$ & $0.008^{b}$ \\
\hline Race & & & & $0.450^{b}$ \\
\hline American Indian or Alaska Native & $2(0.1)$ & $1(0.1)$ & $1(0.4)$ & \\
\hline Asian & $31(1.3)$ & $28(1.3)$ & $3(1.1)$ & \\
\hline Black or African American & $601(24.9)$ & $535(25.0)$ & $66(23.7)$ & \\
\hline White & $1,667(69.1)$ & $1,470(68.8)$ & 197 (70.9) & \\
\hline Unknown & $113(4.7)$ & $102(4.8)$ & $11(4.0)$ & \\
\hline Ethnicity & & & & $0.097^{b}$ \\
\hline Hispanic or Latino & $78(3.2)$ & $73(3.4)$ & $5(1.8)$ & \\
\hline Non-Hispanic or Latino & $2,262(93.7)$ & $2,002(93.7)$ & $260(93.5)$ & \\
\hline Unknown & $74(3.1)$ & $61(2.9)$ & $13(4.7)$ & \\
\hline \multicolumn{5}{|l|}{ Vital sign } \\
\hline First SBP $(\mathrm{mm} \mathrm{Hg})$ & $104(88-127)$ & 104 (89-128) & $98(82-117)$ & $<0.001^{\mathrm{a}}$ \\
\hline Minimum SBP (mm Hg) & 71 (60-81) & 73 (62-82) & $54(42-64)$ & $<0.001^{a}$ \\
\hline Minimum SBP in the ED $(\mathrm{mm} \mathrm{Hg})$ & $81(70-96)$ & $82(71-97)$ & $74(61-88)$ & $<0.001^{\mathrm{a}}$ \\
\hline First MAP $(\mathrm{mm} \mathrm{Hg})$ & $68(58-85)$ & $69(59-86)$ & $66(54-79)$ & $<0.001^{a}$ \\
\hline First heart rate & $107(90-122)$ & $107(90-121)$ & $109(89-126)$ & $0.386^{\mathrm{a}}$ \\
\hline First shock index & $1.0(0.8-1.2)$ & $1.0(0.8-1.2)$ & $1.1(0.9-1.4)$ & $<0.001^{\mathrm{a}}$ \\
\hline First oxygen saturation (\%) & $96(93-99)$ & $96(93-99)$ & $96(91-99)$ & $0.228^{\mathrm{a}}$ \\
\hline Minimum oxygen saturation (\%) & $85(73-90)$ & $86(76-90)$ & $72(53-83)$ & $<0.001^{\mathrm{a}}$ \\
\hline $\begin{array}{l}\text { Minimum oxygen saturation in the emergency } \\
\text { department }(\%)\end{array}$ & 93 (88-96) & 93 (89-96) & 90 (80-94) & $<0.001^{\mathrm{a}}$ \\
\hline First temperature $\left({ }^{\circ} \mathrm{C}\right)$ & $37.0(36.5-38.1)$ & $37.0(36.6-38.2)$ & $36.6(36.1-37.8)$ & $<0.001^{a}$ \\
\hline Maximum temperature $\left({ }^{\circ} \mathrm{C}\right)$ & $38.2(37.4-39.2)$ & $38.3(37.5-39.2)$ & $37.8(36.9-39.0)$ & $<0.001^{\mathrm{a}}$ \\
\hline Maximum temperature in the $\mathrm{ED}\left({ }^{\circ} \mathrm{C}\right)$ & $37.4(36.7-38.7)$ & $37.4(36.7-38.7)$ & $37.1(36.4-38.4)$ & $<0.001^{\mathrm{a}}$ \\
\hline
\end{tabular}


Table 1. Continued

\begin{tabular}{|c|c|c|c|c|}
\hline Factor & Total $(n=2,414)$ & Non-72-hr death $(n=2,136)$ & 72-hr death $(n=278)$ & P-value \\
\hline \multicolumn{5}{|l|}{ Laboratory value } \\
\hline Sodium (mEq/L) & $137(133-140)$ & $137(133-140)$ & $137(133-141)$ & $0.453^{\mathrm{a}}$ \\
\hline Glucose (mg/dl) & $132(105-191)$ & $132(105-190)$ & $131(97-198)$ & $0.530^{\mathrm{a}}$ \\
\hline Creatinine (mg/dl) & $1.5(1.0-2.4)$ & $1.4(1.0-2.3)$ & $2.0(1.3-3.0)$ & $<0.001^{\mathrm{a}}$ \\
\hline Bilirubin (mg/dl) & $0.9(0.6-1.5)$ & $0.9(0.6-1.4)$ & $1.2(0.7-2.2)$ & $<0.001^{\mathrm{a}}$ \\
\hline INR & $1.2(1.1-1.6)$ & $1.2(1.1-1.5)$ & $1.5(1.2-2.1)$ & $<0.001^{\mathrm{a}}$ \\
\hline Albumin $(g / d l)$ & $2.7(2.2-3.2)$ & $2.8(2.3-3.3)$ & $2.4(1.9-2.9)$ & $<0.001^{\mathrm{a}}$ \\
\hline WBC count $\left(\times 10^{9} / \mathrm{L}\right)$ & $13.7(9.0-19.9)$ & $13.7(9.1-19.7)$ & $14.2(8.2-21.9)$ & $0.682^{\mathrm{a}}$ \\
\hline First lactate $(\mathrm{mmol} / \mathrm{L})$ & $4.3(2.4-6.1)$ & $4.2(2.3-5.7)$ & $6.1(3.9-9.4)$ & $<0.001^{\mathrm{a}}$ \\
\hline Maximum lactate (mmol/L) & $4.6(2.8-6.8)$ & $4.4(2.6-6.2)$ & $7.9(5.0-12.5)$ & $<0.001^{\mathrm{a}}$ \\
\hline Maximum lactate in the ED (mmol/L) & $4.5(2.6-6.3)$ & $4.3(2.4-5.9)$ & $6.4(4.5-9.9)$ & $<0.001^{\mathrm{a}}$ \\
\hline \multicolumn{5}{|l|}{ Infection source } \\
\hline Bacteremia & $3(0.1)$ & $3(0.1)$ & 0 & $0.990^{c}$ \\
\hline UTI & $673(27.9)$ & $614(28.7)$ & $59(21.2)$ & $0.009^{b}$ \\
\hline Pneumonia & $716(29.7)$ & $617(28.9)$ & $99(35.6)$ & $0.021^{b}$ \\
\hline Cellulitis & $178(7.4)$ & $164(7.7)$ & $14(5.0)$ & $0.110^{b}$ \\
\hline Clostridium difficile colitis & $133(5.5)$ & $124(5.8)$ & $9(3.2)$ & $0.078^{b}$ \\
\hline MRSA & 165 (6.8) & $140(6.6)$ & $25(9.0)$ & $0.130^{b}$ \\
\hline CRE & $82(3.4)$ & 74 (3.5) & $8(2.9)$ & $0.610^{b}$ \\
\hline \multicolumn{5}{|l|}{ Resuscitation variable } \\
\hline Hour to receive antibiotics & $0.2(0.0-0.5)$ & $0.2(0.0-0.5)$ & $0.2(0.0-0.6)$ & $0.001^{\mathrm{a}}$ \\
\hline Volume in 3 hours (ml/kg) & $40.1(29.7-56.0)$ & $40.0(29.5-55.6)$ & $41.9(31.2-58.4)$ & $0.109^{\mathrm{a}}$ \\
\hline Fluid volume total in 3 hours (ml) & $3,021.5(2,000.0-4,130.0)$ & $3,025.0(2,000.5-4,150.0)$ & $3,000.0(2,000.0-4100.0)$ & $0.606^{\mathrm{a}}$ \\
\hline Volume in 6 hours $(\mathrm{ml} / \mathrm{kg})$ & $47.5(33.4-68.0)$ & $47.2(33.3-67.6)$ & $52.2(36.0-72.2)$ & $0.040^{\mathrm{a}}$ \\
\hline Fluid volume total in 6 hours (ml) & $3,650.0(2,500.0-5,000.0)$ & $3,674.5(2,500.0-5,000.0)$ & $3,590.5(2,400.0-5,150.0)$ & $0.993^{\mathrm{a}}$ \\
\hline Bundle compliance 3 hours & $1,482(61.4)$ & $1,307(61.2)$ & $175(62.9)$ & $0.570^{b}$ \\
\hline Vasopressor use within 6 hours & $1,501(62.2)$ & $1,274(59.6)$ & $227(81.7)$ & $<0.001^{b}$ \\
\hline Intubation & $676(28.0)$ & $521(24.4)$ & $155(55.8)$ & $<0.001^{b}$ \\
\hline Need for renal replacement therapy & $140(5.8)$ & $135(6.3)$ & $5(1.8)$ & $0.002^{b}$ \\
\hline Pulmonary edema within 72 hours & $73(18.0)$ & $68(19.0)$ & $5(10.2)$ & $0.130^{b}$ \\
\hline
\end{tabular}

Values presented as number (\%) or median (interquartile range) or number (\%).

BMI: body mass index; SBP: systolic blood pressure; ED: emergency department; MAP: mean arterial pressure; DNR: do-not-resuscitate; INR: international normalized ratio; WBC: white blood cell; UTI: urinary tract infection; MRSA: methicillin-resistant Staphylococcus aureus; CRE: carbapenem-resistant Enterobacteriaceae.

${ }^{a}$ Kruskal-Wallis test; ${ }^{b}$ Pearson chi-square test; ${ }^{\circ}$ Fisher's exact test.

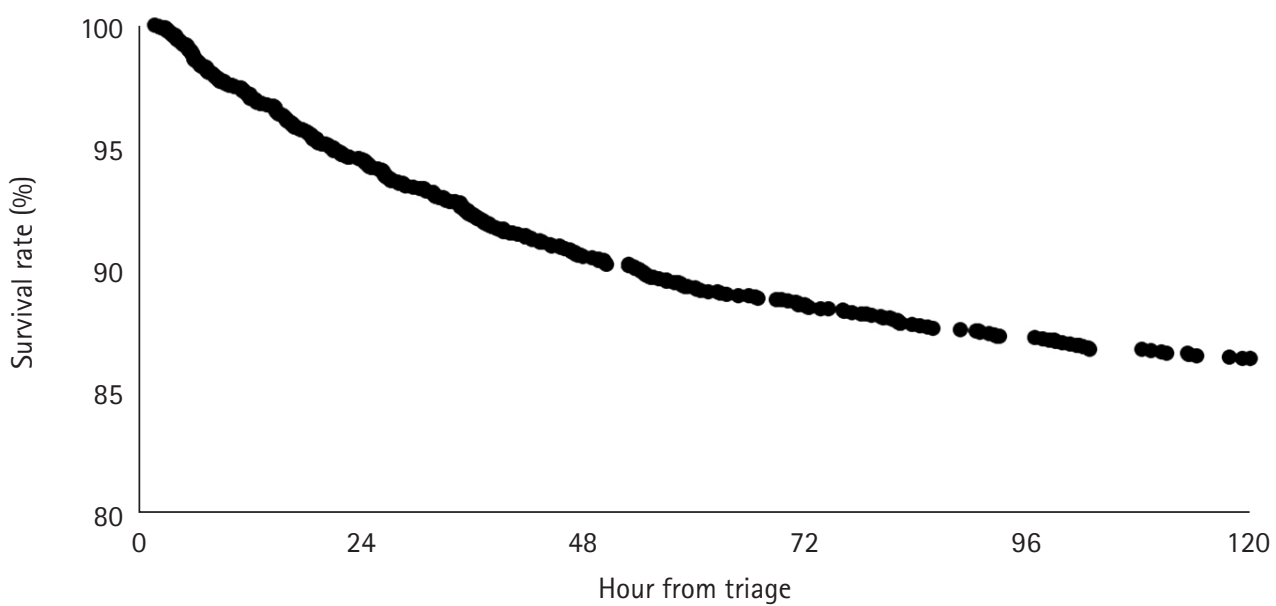

Figure 2. Cumulative survival rate in adults with septic shock relative to time from emergency department triage. 
al curve of our study population.

A number of early clinical variables were different between the outcome groups. Compared to patients who survived beyond 72 hours, patients who died within 72 hours of ED presentation were more likely to be older, have a lower body mass index, have a malignancy, and have dementia. They were also more likely to have pneumonia as the source of sepsis, require early vasopressors, and have a "do not resuscitate" order from admission. Urinary tract infection (UTI) as the source of sepsis was inversely associated with early mortality.

Compared to survivors, patients suffering early mortality were more likely to have the following laboratory findings: higher initial serum creatinine, higher initial serum bilirubin, higher initial international normalized ratio (INR), lower initial albumin, higher initial serum lactate, and higher maximum serum lactate. Physiologic parameters associated with early mortality included: lower initial triage and minimum ED SBP, lower initial triage MAP, higher initial triage SI, lower initial and maximum temperature, and lower minimum oxygen saturation. Among resuscitation variables, increased time to receive antibiotics was associated with higher early mortality. However, there were no differences in volume of resuscitation fluid within 3 hours, 6 hours, or 3-hour bundle compliance between groups.

Based on univariate analysis (Table 2), the following factors were included as candidates for the initial regression model: age, malignancy, dementia, first SBP, minimum SBP, minimum SBP in the ED, first SI, first MAP, UTI as source of sepsis, pneumonia as source of sepsis, vasopressor use within 6 hours, initial creatinine, initial bilirubin, initial albumin, initial INR, first lactate, maximum lactate, and maximum lactate in the ED.

Table 2 describes the multivariate regression analysis model demonstrating the following factors to be independently associated with early septic shock mortality: age (OR, 1.04; 95\% CI, 1.03-1.05), malignancy (OR, 1.53; 95\% CI, 1.11-2.11), pneumonia (OR, 1.39; 95\% CI, 1.02-1.88), UTI (OR, 0.63; 95\% CI, 0.440.89 ), first SI (OR, 1.85; 95\% CI, 1.27-2.70), early vasopressor use (OR, 2.16; 95\% CI, 1.60-2.92), initial INR (OR, 1.14; 95\% CI,

Table 2. Univariate and multivariate analysis

\begin{tabular}{|c|c|c|c|c|}
\hline \multirow{2}{*}{ Domain with variables } & \multicolumn{2}{|c|}{ Univariate } & \multicolumn{2}{|c|}{ Final model (all) } \\
\hline & OR (95\% Cl) & P-value & OR $(95 \% \mathrm{Cl})$ & P-value \\
\hline \multicolumn{5}{|l|}{ Demographics } \\
\hline Age & $1.03(1.02-1.04)$ & $<0.01$ & $1.04(1.03-1.05)$ & $<0.01$ \\
\hline Dementia & $1.39(1.04-1.86)$ & 0.04 & & \\
\hline Malignancy & $1.9(1.45-2.51)$ & $<0.01$ & $1.53(1.11-2.11)$ & $<0.01$ \\
\hline \multicolumn{5}{|l|}{ Hemodynamics } \\
\hline First SBP & $0.99(0.99-1.00)$ & $<0.01$ & & \\
\hline Min SBP & $0.93(0.92-0.94)$ & $<0.01$ & & \\
\hline Min SBP in ED & $0.98(0.97-0.99)$ & $<0.01$ & & \\
\hline First shock index & $2.35(1.69-3.27)$ & $<0.01$ & $1.85(1.27-2.70)$ & 0.04 \\
\hline First MAP & $0.99(0.98-1.00)$ & $<0.01$ & & \\
\hline \multicolumn{5}{|l|}{ Source of infection } \\
\hline UTI & $0.67(0.50-0.91)$ & 0.01 & $0.63(0.44-0.89)$ & $<0.01$ \\
\hline Pneumonia & $1.37(1.05-1.78)$ & 0.03 & $1.39(1.02-1.88)$ & 0.03 \\
\hline \multicolumn{5}{|l|}{ Intervention } \\
\hline Vasopressor within 6 hours & $2.47(1.91-3.20)$ & $<0.01$ & $2.16(1.60-2.92)$ & $<0.01$ \\
\hline \multicolumn{5}{|l|}{ Laboratory value } \\
\hline Creatinine & $1.07(1.02-1.13)$ & 0.01 & & \\
\hline Bilirubin & $1.09(1.05-1.13)$ & $<0.01$ & & \\
\hline Albumin & $0.49(0.41-0.59)$ & $<0.01$ & $0.55(0.44-0.69)$ & $<0.01$ \\
\hline INR & $1.24(1.16-1.34)$ & $<0.01$ & $1.14(1.07-1.27)$ & $<0.01$ \\
\hline First lactate & $1.22(1.18-1.27)$ & $<0.01$ & $1.21(1.16-1.26)$ & $<0.01$ \\
\hline Max lactate & $1.27(1.23-1.31)$ & $<0.01$ & & \\
\hline Max lactate in ED & $1.23(1.19-1.28)$ & $<0.01$ & & \\
\hline
\end{tabular}

OR: odds ratio; Cl: confidence interval; SBP: systolic blood pressure; ED: emergency department; MAP: mean arterial pressure; UTI: urinary tract infection; INR: international normalized ratio. 
1.07-1.27), initial albumin (OR, 0.55; 95\% CI, 0.44-0.69), and first serum lactate (OR, 1.21; 95\% CI, 1.16-1.26).

The optimal cut point for initial serum lactate level for prediction of early morality was $4.89 \mathrm{mmol} / \mathrm{L}$, with AUC of 0.693, sensitivity of 0.669 , and specificity of 0.651 . For initial SI, the optimal cut point was 1.13, with AUC of 0.573 , sensitivity of 0.468 , and specificity of 0.672 (Figure 3 ).

\section{DISCUSSION}

This is the largest known study evaluating early mortality of patients presenting with acute septic shock and provides several insights into this disease. Our data demonstrate high early mortality, with approximately one in nine ED septic shock patients dying within 72 hours of presentation. Our 24-hour mortality rate of $5.5 \%$ is consistent with a former study demonstrating $4.9 \%$ mortality within the same time period [4]. Our 72-hour rate is slightly lower than the $14.4 \%$ 72-hour mortality rate found by Daviaud et al [5]. For comparison, the mortality rate of ST segment elevation myocardial infarction patients undergoing percutaneous coronary intervention is comparatively low at 3.4\% at 7 days [6]. These data highlight the disproportionate early mortality associated with acute septic shock with compounded economic and societal impact due to the high prevalence of sepsis [7].

High early septic shock mortality highlights a therapeutic opportunity if this subpopulation is recognized early, which brings us to the next study aim of early identification of highrisk patients. We identified nine discriminating clinical variables among ED patients with septic shock that were independently associated with early death.

As expected, older age is associated with increased risk of early mortality in sepsis. Older patients tend to have more co-morbid medical conditions and less physiologic reserve, decreasing their ability to recover from critical illnesses [8]. Similar to our finding that malignancy increases risk of early mortality, in a large study of over 1.7 million cancer patients with sepsis published in 2006, cancer was found to be an independent predictor of mortality [9]. The results of the present study show that patients with underlying malignancy are 1.53 times more likely to die within 72 hours of presentation than those without. We propose several potential explanations for this association, including attenuated immune response and immunomodulating treatments leading to rapidly overwhelming infection, and higher rate of early limitation or withdrawal of aggressive care. Although "do not resuscitate" directive status was associated with increased early mortality in univariate analysis, this association did not persist in multivariate analysis, which reinforces the finding that other patient factors were more important than directives code status.

Among our cohort, pneumonia was both the most common source of infection and the only source associated with
(A) Initial serum lactate cut point $4.89 \mathrm{mmol} / \mathrm{L}$

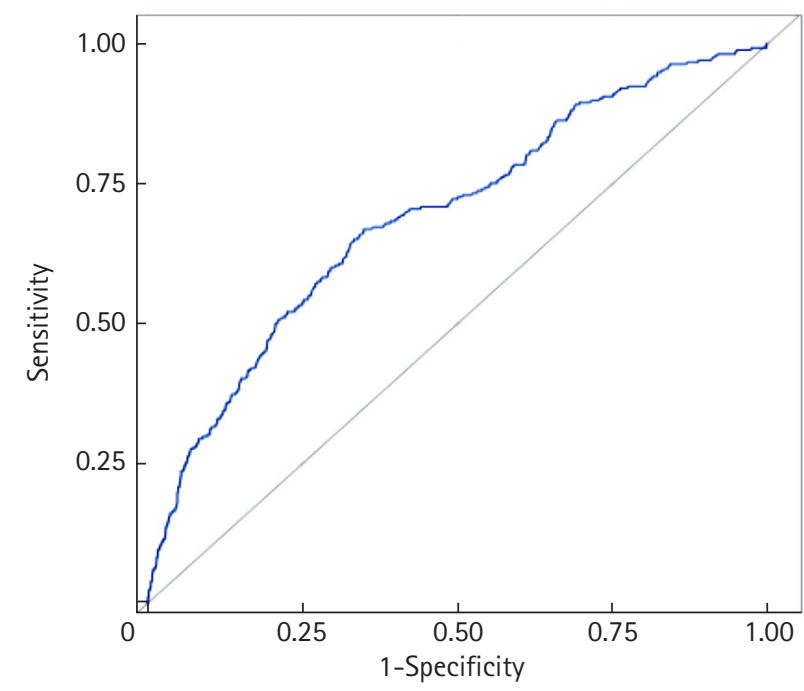

(B) Initial shock index cut point 1.13

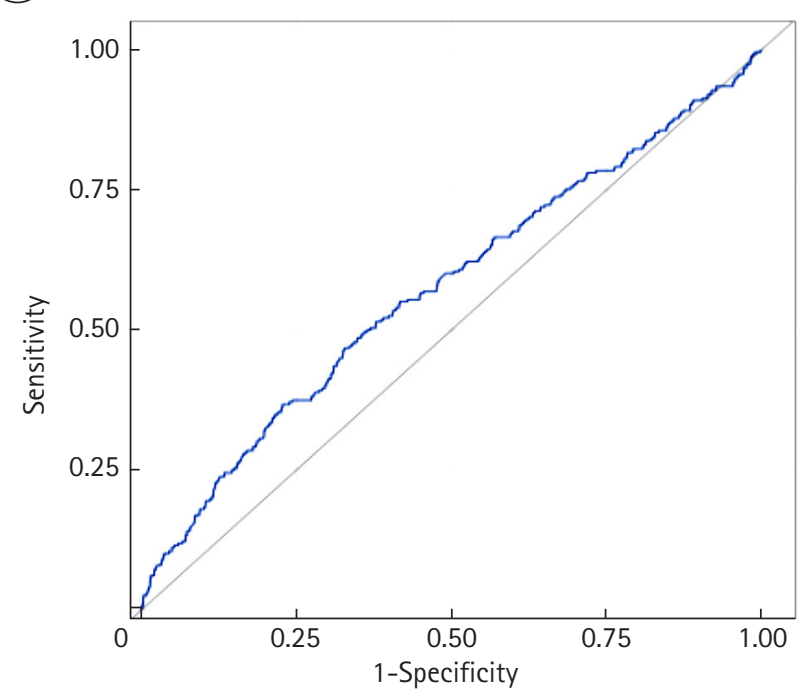

Figure 3. Receiver operating curves (ROC) for prediction of early mortality. (A) ROC for initial serum lactate. (B) ROC for initial shock index. AUC: area under the curve. 
increased early sepsis mortality. This finding reinforces prior work and is an important clinical reminder of pneumonia lethality and the need for heightened vigilance in screening of pneumonia patients for early markers of organ dysfunction and complicated disease progression [10,11]. In contrast, UTI as the source of sepsis was associated with a lower risk of early mortality and may highlight an opportunity for successful de-escalation of critical care resources once these patients show evidence of clinical improvement.

Presenting physiologic variables were important objective markers of lethal septic shock. Regression analysis demonstrated best fit with presentation SI compared to first MAP or first SBP. This is consistent with prior literature showing that SI is an independent predictor of 28-day mortality [12,13]. Based on the ROC for initial SI, a cut point of 1.13 provided optimal sensitivity and specificity and can be a quick risk stratification tool for early mortality. Similarly, hypotension requiring vasopressor therapy within the first 6 hours of presentation was also associated with increased early mortality, furthering the concept that fluid resistant shock is a clear marker of severe illness.

Serum lactate level is a well-described marker of illness severity and is currently used for risk stratification in sepsis. In many studies it has been shown to be a predictor of mortality in sepsis $[4,5,14,15]$. The results of this study confirm that lactate is also a predictor of early mortality. The AUC for initial lactate of 0.693 is consistent with the findings of a recent study showing an AUC for initial lactate of 0.70 for prediction of 28-day mortality [16]. Based on our data, a cut point of 4.89 $\mathrm{mmol} / \mathrm{L}$ is a useful threshold for risk stratification of patients at high risk of early death.

Three other laboratory values, INR, bilirubin, and albumin, were found to be predictors of early mortality that were not previously identified in the literature. Albumin was found to be inversely related to early mortality, meaning a lower albumin level was associated with a higher risk of early mortality. These three values likely correlate with concomitant liver dysfunction, which is a known predictor of mortality in all critically ill patients [17].

Time to initiation of antibiotics was associated with early mortality in univariate analysis but did not persist in the multivariate model. ED resuscitation fluid volume and 3-hour bundle compliance were not significantly different between groups. This is of particular interest given the large emphasis placed on sepsis bundles. We suspect that overall bundle compliance, including initial antibiotic timeliness, was sufficient and clinically similar enough that it was not an independent factor associated with our outcome. For reference, the overall bundle compliance in our study group was $63 \%$, which is higher than the reported national average of $49 \%$ [18].

The evolving paradigm of sepsis justifiably highlights it as a highly time-dependent disease. Our institutional pathway focuses on early identification and standardized treatment bundles aimed to expedite early standardized resuscitation. Our study data add to this approach by providing additional clinical criteria to categorize patients at risk of early death and potentially eligible for additional interrogation and treatments to improve outcomes.

The signal of greater cardiovascular failure manifested by greater SI and early vasopressor use in the early mortality group highlights an opportunity for improved early hemodynamic characterization and manipulation as one potential opportunity. While resuscitation volume was similar in the two groups, this does not imply adequacy of fluid resuscitation and under-resuscitation of patients with severe cardiovascular derangement may be identifiable by early hemodynamic assessment [19]. Using additional monitoring techniques, including microperfusion assessment, is another possible way to target future sepsis treatments in this high risk population [20].

New treatment strategies for sepsis have achieved variable levels of success. This variable success may be due to the wide spectrum of severity in patients currently defined as having sepsis. In this study, we highlight objective criteria to help identify high-risk patients who might benefit most from early adjunctive therapies.

This study comes with several limitations. Our retrospective design is associated with potential biases and inaccuracies in data. We utilized a dedicated patient registry of patients managed via a standardized treatment pathway in attempt to minimize the impact of these issues. Additionally, chart abstraction was performed with a standardized data collection tool. Recognizing that our clinical pathway registry may have included patients initially incorrectly diagnosed with sepsis, we specifically excluded patients based on the absence of confirmed infection based on discharge diagnosis coding. Traditional mortality predictors such as Sequential Organ Failure Assessment (SOFA) and Acute Physiologic Assessment and Chronic Health Evaluation (APACHE) were not available from our database and were therefore not included for comparative analysis, however a recent study by Liu et al found that serum lactate level in septic patients was similar to SOFA score as a predictor of mortality [21]. The clinical definition used for en- 
try into our high-risk sepsis ED pathway may vary from newer sepsis consensus definitions, which may limit comparisons to other populations. Patients who died within 72 hours were not directly compared with those who died after 72 hours. However, this is an area of potential future research as there may be important differences or commonalities between these two groups. Lastly, our database did not include information necessary to ascertain the clinical analysis associated with death, such as discrimination of sudden unexpected death versus withdrawal of care in the context of deteriorating clinical status or comorbidity burden. Septic shock patients experience a high rate of early mortality within 72 hours of ED arrival. Recognizable factors may aid in identification of patients at risk of early death who may benefit from additional early monitoring and therapies.

\section{CONFLICT OF INTEREST}

No potential conflict of interest relevant to this article was reported.

\section{ACKNOWLEDGMENTS}

The abstract was presented at the Society of Academic Emergency Medicine annual meeting in Indianapolis, IN, USA, on May 16, 2018.

\section{ORCID}

Matthew S. Reaven

https://orcid.org/0000-0003-2279-0974

Nigel L. Rozario https://orcid.org/0000-0003-1510-1675

Maggie S. J. McCarter

Alan C. Heffner https://orcid.org/0000-0002-5169-9363 https://orcid.org/0000-0002-0438-4991

\section{AUTHOR CONTRIBUTIONS}

Conceptualization: MSR, ACH. Data curation: NLR. Formal analysis: NLR, MSJM. Methodology: MSR, ACH. Project administration: ACH. Writing-original draft: MSR,. Writing-review \& editing: NLR, MSJM, ACH.

\section{REFERENCES}

1. Gupta RG, Hartigan SM, Kashiouris MG, Sessler CN, Bearman GM. Early goal-directed resuscitation of patients with septic shock: current evidence and future directions. Crit Care
2015;19:286

2. Angus DC, van der Poll T. Severe sepsis and septic shock. N Engl J Med 2013;369:840-51.

3. Winters BD, Eberlein M, Leung J, Needham DM, Pronovost PJ, Sevransky JE. Long-term mortality and quality of life in sepsis: a systematic review. Crit Care Med 2010;38:1276-83.

4. Javed A, Guirgis FW, Sterling SA, Puskarich MA, Bowman J, Robinson T, et al. Clinical predictors of early death from sepsis. J Crit Care 2017;42:30-4.

5. Daviaud F, Grimaldi D, Dechartres A, Charpentier J, Geri G, Marin N, et al. Timing and causes of death in septic shock. Ann Intensive Care 2015;5:16.

6. Doost Hosseiny A, Moloi S, Chandrasekhar J, Farshid A. Mortality pattern and cause of death in a long-term follow-up of patients with STEMI treated with primary PCI. Open Heart 2016;3:e000405

7. Paoli CJ, Reynolds MA, Sinha M, Gitlin M, Crouser E. Epidemiology and costs of sepsis in the united states-an analysis based on timing of diagnosis and severity level. Crit Care Med 2018;46:1889-97.

8. Haas B, Wunsch H. How does prior health status (age, comorbidities and frailty) determine critical illness and outcome? Curr Opin Crit Care 2016;22:500-5.

9. Danai PA, Moss M, Mannino DM, Martin GS. The epidemiology of sepsis in patients with malignancy. Chest 2006;129:1432-40.

10. He XL, Liao XL, Xie ZC, Han L, Yang XL, Kang Y. Pulmonary infection is an independent risk factor for long-term mortality and quality of life for sepsis patients. Biomed Res Int 2016;2016:4213712.

11. Honselmann KC, Buthut F, Heuwer B, Karadag S, Sayk F, Kurowski V, et al. Long-term mortality and quality of life in intensive care patients treated for pneumonia and/or sepsis: Predictors of mortality and quality of life in patients with sepsis/ pneumonia. J Crit Care 2015;30:721-6.

12. Tseng J, Nugent K. Utility of the shock index in patients with sepsis. Am J Med Sci 2015;349:531-5.

13. Heffner AC, Swords DS, Neale MN, Jones AE. Incidence and factors associated with cardiac arrest complicating emergency airway management. Resuscitation 2013;84:1500-4.

14. Shapiro NI, Howell MD, Talmor D, Nathanson LA, Lisbon A, Wolfe RE, et al. Serum lactate as a predictor of mortality in emergency department patients with infection. Ann Emerg Med 2005;45:524-8.

15. Mikkelsen ME, Miltiades AN, Gaieski DF, Goyal M, Fuchs BD, Shah CV, et al. Serum lactate is associated with mortality in severe sepsis independent of organ failure and shock. Crit Care 
Med 2009;37:1670-7.

16. Ryoo SM, Lee J, Lee YS, Lee JH, Lim KS, Huh JW, et al. Lactate level versus lactate clearance for predicting mortality in patients with septic shock defined by sepsis-3. Crit Care Med 2018;46:e489-95.

17. Horvatits T, Drolz A, Trauner M, Fuhrmann V. Liver injury and failure in critical illness. Hepatology 2019;70:2204-15.

18. Barbash IJ, Davis B, Kahn JM. National performance on the Medicare SEP-1 sepsis quality measure. Crit Care Med 2019; 47:1026-32.

19. Nowak RM, Nanayakkara P, DiSomma S, Levy P, Schrijver E,
Huyghe R, et al. Noninvasive hemodynamic monitoring in emergency patients with suspected heart failure, sepsis and stroke: the PREMIUM registry. West J Emerg Med 2014;15:78694.

20. De Backer D, Donadello K. Assessment of microperfusion in sepsis. Minerva Anestesiol 2015;81:533-40.

21. Liu Z, Meng Z, Li Y, Zhao J, Wu S, Gou S, et al. Prognostic accuracy of the serum lactate level, the SOFA score and the qSOFA score for mortality among adults with Sepsis. Scand J Trauma Resusc Emerg Med 2019;27:51. 